\title{
6 \\ Los sistemas de Fiscalización y Control \\ en las Cooperativas: aplicación al caso de Costa Rica
}

\author{
Oscar Alonso Hernández Vargas \\ Isaías López Lara
}

\section{Introducción}

El Estado costarricense mediante el Instituto de Fomento del Cooperativismo fomenta, promueve, financia, divulga y apoya el cooperativismo en todos los niveles, propiciando las condiciones requeridas y los elementos indispensables, a una mayor y efectiva participación de la población del país, en el desenvolvimiento de la actividad económico-social que simultáneamente contribuya a crear mejores condiciones de vida para los habitantes de escasos recursos, realizar una verdadera promoción del hombre costarricense y fortalecer la cultura democrática nacional».

También el Estado mediante la Superintendencia General de Entidades Financieras supervisa lo que son las cooperativas de ahorro y crédito, a raíz de la crisis que sufrió el sector cooperativo, como la desencadenada a raíz de la problemática surgida en COOVIVIENDA. Esta situación puso en peligro no sólo a las cooperativas del sector, sino a cooperativas de todos los sectores que de manera directa o indirecta son propietarias, han tenido inversiones o relaciones con los bancos y entidades financieras cooperativas que entraron en crisis. Es decir, la situación trasciende del sector donde se origina el problema y amenaza la estabilidad del sistema cooperativo como un todo.

En este sentido, se realizó investigación con el propósito de mostrar el nivel de intervención del Estado costarricense en el sector cooperativo, referida a los Sistemas de Fiscalización y Control en las Cooperativas y Supervisión de las Cooperativas de Ahorro y Crédito por parte de la Superintendencia General de Entidades Financieras. 
Dicha investigación se estructuró primeramente con un análisis de los sistemas de fiscalización y control de cooperativas y luego una descripción del grado de supervisión que se realiza en las cooperativas de ahorro y crédito en nuestro país.

\section{Los sistemas de Fiscalización y Control en las Cooperativas: aplicación al caso de Costa Rica}

\section{Antecedentes}

Las organizaciones cooperativas surgen conforme a la necesidad social existente en un momento histórico determinado, por lograr una mayor unidad organizativa y laboral en razón de una mejor producción y por ende mejores resultados, ya sea en la actividad efectuada o en el producto final que se logra. La idea cooperativa se oriento en una participación de los individuos con su fuerza laboral y económica en virtud de lograr mejores resultados para sí mismos y una repartición equitativa de los dividendos obtenidos por el esfuerzo.

Dicha concepción de solidaridad y dividendo resultaba muy simple de aplicar en un principio, pues enfocaba una forma de organización social dirigida a la producción de beneficios para sus miembros, pero con el paso del tiempo y la extensión en los campos de aplicación de este tipo de organizaciones, empiezan a ser necesarios el establecimiento de mecanismos de control, tanto hacia adentro como hacia fuera, con el fin de procurar la mayor claridad de los entes cooperativos, ya sea con sus asociados y el respectivo respeto a la posición de cada uno, como también el conservar que la actividad realizada siga dando los réditos deseados en armonía con todos los mecanismos de regulación vigentes para la misma; sin desmeritar la esencia contemplada por la organización cooperativa.

Se concibe a las cooperativas como un ente de derecho privado con una organización autónoma, producto de la alianza de grupos de trabajadores con objetivos en común y en respuesta a las necesidades inmediatas de los mismos. A la vez se entiende a estos entes como fuentes creadoras de empleo, lo que también respalda la función de colchón social que se esgrime de estas instituciones y su función independiente de la actividad estatal directa y aparte a la estructura gubernamental. 
Como parte de la evolución natural del ente cooperativo no podemos negar la existencia de un nexo importante con la actividad estatal, tanto en los fines sociales que ambos persiguen como en los objetos que desarrollan, además que hoy en día, en todos los países del mundo en que existe una actividad cooperativa activa el Estado ejerce fiscalización sobre su funcionamiento. Claro está, no en todos los países la fiscalización se ejerce de la misma forma, ni con el mismo grado de eficacia, pero sí sigue siendo un elemento presente dentro de la función gubernamental de control.

\section{Naturaleza jurídica}

Cabe señalar a las entidades cooperativas en cuanto a su naturaleza jurídica, como sujetos de derecho privado, con una personalidad jurídica propia, la cual les permite negociar directamente y bajo su propia responsabilidad. Pero a su vez en el caso de Costa Rica, con todo y esta autonomía que dichas organizaciones sociales poseen, existen ciertas instancias como el CONACOOP (Consejo Nacional de Cooperativas) y el mismo INFOCOOP (Instituto Nacional de Fomento Cooperativo) que ejercen un control previo y directo sobre todos los entes cooperativos y la actividad que estos efectúan. Control que se ejecuta de forma a priori, y no con base al tipo de la actividad o la especialidad del campo en que se desarrolla, sino que el control se efectúa por el hecho de ser cooperativas, lo cual es suficiente elemento para que estas organizaciones fiscalicen la estructura y el desarrollo de sus asociados.

Como una de las consecuencias de esa relación Estado-Cooperativismo, pese al origen de entidades de Derecho Privado de las Cooperativas, se inició la costumbre de introducir en las normas jurídicocooperativas, ciertas disposiciones dirigidas a legitimar el control estatal dentro de estas organizaciones. Y los ejemplos son abundantes y parecen ser parte del Derecho Natural Cooperativo, como lo podemos ver en Latinoamérica, en la Ley Marco de Cooperativas de América, dentro del cual la organización de Cooperativas de América, introduce como un aparte importante lo que se denomina la Autoridad de Aplicación. ${ }^{1}$

1 SÁnchez BozA, Roxana. Tendencias a nivel internacional sobre los sistemas de fiscalización y control de las cooperativas. Documento Inédito. 
El Estado procura ubicar a las entidades cooperativas dentro del régimen jurídico-legal vigente, y lo hace, asimilándolas a otras organizaciones ya legisladas, o bien sancionando a su respecto leyes y otras disposiciones especiales mas o menos amplias.

\section{Primeros indicios fiscalizadores en la legislación cooperativa}

En las primeras épocas de su desarrollo, las cooperativas frecuentemente se adaptaron a las leyes vigentes sobre diversas asociaciones civiles o sobre sociedades mercantiles. Sin embargo, el crecimiento de las entidades cooperativas y el estrechamiento de sus relaciones con las demás personas físicas y jurídicas, inducen al Estado a dictar disposiciones legales específicas, mediante las cuales tanto el Estado como los demás sujetos del ordenamiento jurídico diferencian a las cooperativas de las demás entidades, estableciendo los caracteres de su organización y funcionamiento ${ }^{2}$, procurando defender los derechos de los asociados y de los terceros que contratan con ellos, así como los lineamientos que determinan las formas de fiscalización.

Paulatinamente y con el desarrollo del movimiento cooperativo se empezaron a dictar disposiciones cooperativas en distintos países incluidas o no dentro de los códigos civiles, comerciales u otros cuerpos legales. Pero, en la actualidad algunos países de gran desarrollo cooperativo poseen leyes completas y modernas sobre la materia.

En los países de mayor tradición cooperativa, las normas de organización y funcionamiento de las entidades surgen y se aplican espontáneamente con base a las experiencias realizadas; luego las federaciones $u$ otras asociaciones de cooperativas adoptan tales normas fundamentales en los estatutos. Por el contrario en los países de menor desarrollo cooperativo, la legislación y por ende la fiscalización suele anticiparse a la práctica del cooperativismo y adopta a menudo un detallado ordenamiento legal que toma como modelo las normas aplicadas en los países donde las cooperativas se hallan más desarrolladas, facilitando la difusión de este tipo de entidades 3 .

2 KAPLAN, Alicia y DRIMER, Bernardo. Las Cooperativas: Fundamentos - Historia Doctrina. Argentina 1981. 3. ${ }^{a}$ edición.

3 Kaplan, Alicia y Drimer, Bernardo. Las Cooperativas: Fundamentos - Historia Doctrina. Argentina 1981. 3. ${ }^{a}$ edición. 


\section{Sistema de Fiscalización Cooperativa aplicado en Costa Rica}

Costa Rica posee un sistema de fiscalización cooperativa que intenta actuar de manera preventiva y de manera tal que se refleje tanto un control a lo interno del sector cooperativo mediante instituciones como el Consejo Nacional de Cooperativas, cuyos órganos agrupan la participación de todas las cooperativas asociadas del país y además orientan y coordinan la actividad cooperativa con otras instituciones sociales y el propio Estado. Dándole de está forma una representación más sólida al sector cooperativo, con el fin de alcanzar los fines propuestos por los mismos. El Consejo Nacional de Cooperativas es un órgano colegiado de representantes del cooperativismo costarricense, creado por Ley en 1973, donde todas las cooperativas del país están asociadas. Realiza su labor en coordinación con las regiones y sectores productivos a través de la consulta permanente a las bases cooperativas con el fin de garantizar el desarrollo armónico del Movimiento.

Es la instancia de representación de mayor jerarquía en el Movimiento Cooperativo, que define e impulsa políticas y estrategias en materia de promoción y planificación del desarrollo cooperativo y articulación de las organizaciones sociales en el ámbito de la economía social. ${ }^{4}$

Por otra parte el Estado creó una institución como el Instituto Nacional de Fomento Cooperativo (INFOCOOP) la cual es una institución pública con personalidad jurídica, patrimonio propio y con autonomía administrativa y funcional, creada para fomentar, promover, financiar, divulgar y apoyar el cooperativismo, fortaleciendo el desarrollo socioeconómico del país.

El Estado al promulgar la ley de creación del INFOCOOP, crea un instrumento para cumplir con mandatos constitucionales como son, por una parte el que dispone el artículo 64 de la Constitución Política ${ }^{5}$ de fomentar el cooperativismo como un medio para mejorar la calidad de vida de los trabajadores y por otra parte, con el contenido de su artículo 50 que le impone el deber de procurar un mayor bienestar a todos los ciudadanos del país, organizando y estimulando la producción y el más adecuado reparto de la riqueza.

4 www.infocoop.go.cr.

5 Constitución Política de la República de Costa Rica. Art. 64. «El Estado fomentará la creación de Cooperativas como medio de facilitar mejores condiciones de vida a los trabajadores.» 
De aquí deriva la importancia que se le otorga a las organizaciones cooperativas como medio de asegurar el bienestar general y el adecuado desarrollo económico-social. A la vez que se refleja la obligación del Estado de fomentar la organización y el funcionamiento de estas entidades.

Por este medio el Estado busca brindar los servicios de asistencia técnica, asesoría, capacitación, supervisión, financiamiento y promoción en el ámbito regional y sectorial, facilitando el desarrollo cooperativo y con alto impacto en la base cooperativa. Además de velar porque las organizaciones cooperativas funcionen ajustadas a la normativa legal vigente, mediante mecanismos de fiscalización apropiados. ${ }^{6}$

Es decir, el Estado intenta brindar una supervisión general de los entes cooperativos y la actividad que éstos efectúan de manera tal que no se restrinja de manera alguna la actividad productiva de los mismos pero buscando a la vez preservar todos los principios cooperativos y el sentido social que tutelan el quehacer de cualquier ente de esta naturaleza. Por lo demás es consecuente señalar que un Estado participativo y solidario con la actividad cooperativa genera beneficios para todos, pues de esta forma redistribuye sus cargas de trabajo e incluso logra una mayor recaudación fiscal con base al crecimiento productivo que puedan experimentar todo este tipo de organizaciones sociales.

A la vez la fiscalización general que realiza el Estado sobre el sector cooperativo por su misma naturaleza, debe orientarse como una medida preventiva no sólo sobre la estructura y formación de las cooperativas, sino más bien, la tutela se debe considerar sobre la actividad y el campo de acción en que se desarrolla dicho ente. En razón de esta necesidad, es que el INFOCOOP ejerce una vigilancia técnica, de forma tal que los lineamientos y la actividad cooperativa se ajusten al marco jurídico vigente sobre el cual deben implementarse, evitando así cualquier disyuntiva jurídica que de este proceso de inserción resulte. ${ }^{7}$

6 www.infocoop.go.cr.

7 Un claro ejemplo de este proceso de vigilancia se puede observar en el Artículo 97 de la Ley de Asociaciones Cooperativas y creación del Instituto Nacional de Fomento Cooperativo, No. 6756 del 5 de mayo de 1982, el cual textualmente dice: «Corresponderá el INFOCOOP, llevar a cabo la más estricta vigilancia sobre las asociaciones cooperativas, con el exclusivo propósito de que éstas funcionen ajustadas a las disposiciones legales. Al efecto, permitirán la inscripción y vigilancia que sus funcionarios practiquen en dichas asociaciones para cerciorarse del cumplimiento de esta ley, de sus reglamentos y leyes conexas y darles la información indispensable que con ese objeto soliciten.» 
Es decir, sería iluso pensar que las cooperativas de ahorro y crédito que hoy en día están sometidas a los controles de intermediación financiera deban de ser tratadas de la misma forma que una cooperativa orientada a la producción de cualquier producto específico por ejemplo el café. Ambas cooperativas pueden presentar un diagnóstico similar, pero el efecto social y perjuicio económico que se puede ocasionar en una cooperativa crediticia y de actividad financiera, en teoría es sobre evaluado, al afectar esta actividad otras esferas jurídicas mayores y tuteladas con mayor severidad por el ordenamiento jurídico, como la buena fe en los negocios o la confianza obligacional derivada del pago de las obligaciones adquiridas.

Esto no desmiente que tal vez el problema sea el mismo e incluso que el efecto de pérdida económica en la cooperativa de producción sea mayor, pero el entorno social juzga de una manera mas drástica el que una institución incumpla con sus inversionistas, quienes son en alguna medida los que han salvaguardado la actividad cooperativa con la confianza en la institución señalada.

La existencia de los controles a lo externo de las cooperativas pero a la vez a lo interno de su actividad, bien se complementa con el status de ejemplo de Democracia con el cual se conoce a las Cooperativas. Y es que estos entes son reflejo de la aplicación de la libertad responsable de los socios aplicada a la solidaridad y el bienestar social, razón suficiente para considerar a las cooperativas como instituciones de formación integral para el ser humano.

Es por estas razones entre otras, que la fiscalización cooperativa inicia desde que se instauran los controles internos en las cooperativas. En cada cooperativa existe un Comité de Vigilancia o de Supervisión ${ }^{8}$ el cual actúa como un filtro sobre el desarrollo de la actividad cooperativa, de manera tal, que son los mismos socios los que ejercen

8 El Artículo 49 de la Ley de Asociaciones Cooperativas y creación del Instituto Nacional de Fomento Cooperativo, No. 6756 del 5 de mayo de 1982, esgrime lo siguiente: Corresponde al comité de vigilancia elegido por la asamblea, que se integrará con un número no menor de tres asociados, el examen y fiscalización de todas las cuentas y operaciones realizadas por la cooperativa, e informar lo que corresponda ante la asamblea. La responsabilidad solidaria de los miembros del consejo de administración y del gerente alcanza a los miembros del comité de vigilancia por los actos que éste no hubiere objetado oportunamente. Quedan exentos de responsabilidad los miembros del comité que salven expresamente su voto dentro del mes siguiente al acto de tomarse la decisión respectiva. 
control de sus propios actos y los que tienen una responsabilidad directa sobre los efectos derivados de los mismos.

Con la misma cooperativa existe la capacidad de que los otros órganos sociales ejerzan sus propios mecanismos de autocontrol tanto en cuanto a la producción como en lo que respecta a los resultados de la misma. Además existe la posibilidad de implementar controles por medio de otros órganos sociales de la propia cooperativa como el mismo Consejo de Administración, a la vez que se refleja también la variable de contratar tanto Auditorías Internas como Externas, por las cuales se cuantifique la situación real en el Balance de la Cooperativa y se evalúe la situación de los socios en cuanto a aportes y resultados obtenidos.

Cabe señalar a la vez, la posibilidad de establecer organismos auxiliares de las Cooperativas con el fin específico de convertirse en un apoyo en cuanto a la Fiscalización Cooperativa, de modo tal que dichos organismos que ostentan personalidad jurídica propia y tienen una duración ya sea definida o indefinida según lo acuerden sus integrantes, ejerzan un control directo en cuanto a la actividad desarrollada por estas cooperativas en específico, tanto en el desarrollo de nuevas actividades como en la expansión de sus mismas operaciones contribuyendo al crecimiento de este sector. ${ }^{9}$

Es importante determinar que la función de fiscalización en las Cooperativas responde a la necesidad de comprobar el cumplimiento de las disposiciones legales, reglamentarias y estatutarias, verificando la consecución de las finalidades previstas.

Dicha función de fiscalización también se manifiesta de manera interna, entre otras actividades, mediante la recepción de las comunicaciones y documentos relativos a la convocatoria a la Asamblea de las Cooperativas, la posibilidad de inspección sobre las actividades, el estudio de los respectivos balances, la realización de comprobaciones especiales y la aplicación en determinados casos de multas u otras sanciones. ${ }^{10}$

En Costa Rica el control ejercido por la Administración Pública con respecto a la actividad desarrollada a lo interno de las cooperativas se ha estancado, como consecuencia del mismo letargo en que ha caído

9 Chaves Gómez, Alvaro. Los Organismos Auxiliares del Movimiento Cooperativo. CEDAL 1985. Costa Rica.

10 Kaplan, Alicia y Drimer, Bernardo. Las Cooperativas: Fundamentos - Historia Doctrina. Argentina 1981. 3. ${ }^{a}$ edición. 
el propio movimiento cooperativo en los últimos tiempos y que obedece principalmente a dos razones específicas como:

- la interiorización de las políticas neoliberales en la mayoría de los países del mundo, razón que ha ocasionado un cambio grande en la concepción de las organizaciones sociales, las cuales hoy en día se ven envueltas en una lucha directa y frontal con las tendencias de corte liberal, basadas casi exclusivamente en indicadores económicos y cuyo objeto es una minimización del aparato estatal que conlleva, a la privatización de sus servicios y a la disminución de la responsabilidad estatal para con los sectores más necesitados.

- la otra razón no se aparta en mucho de este mismo fenómeno mundial expuesto anteriormente, pero refiere ante todo a la disminución de la actividad cooperativa como forma de organización social en beneficio del surgimiento de otras formas de organización con mecanismos más versátiles para el desarrollo de la actividad comercial y que en teoría brindan un mayor beneficio a sus asociados.

El que se den cada vez y de forma más marcada estos elementos, ha ocasionado la disminución real del movimiento cooperativo y por ende, el Estado ha ido perdiendo el interés en el Control cooperativo y ha enfocado su supervisión en otros sectores donde hoy en día se ve más involucrado el interés público como tal.

Enfocando principalmente la fiscalización estatal sobre la actividad cooperativa de ahorro y crédito, sobre la que sí se orientan verdaderos mecanismos de intermediación financiera, dirigidos principalmente para el caso de Costa Rica por parte de la SUGEF (Superintendencia General de Entidades Financieras).

Dicho órgano de Supervisión Estatal se enmarca como una institución especializada en la intervención y fiscalización de entidades financieras con el fin de garantizar la relativa seguridad de la inversión y minimizar los riesgos existentes, por medio de la evaluación periódica de todas las instituciones que manejan fondos ajenos, realizando operaciones de captación de recursos y redistribución de los mismos. Esta Superintendencia actúa como una calificadora de Riesgo para estas instituciones, además de tener la posibilidad de limitar la actividad financiera de las mismas, a la vez que puede emitir directrices y recomendaciones de acatamiento obligatorio para todas las entidades que operan bajo su supervisión. 


\section{Supervisión de las Cooperativas de Ahorro y Crédito por parte de la Superintendencia General de Entidades Financieras Costa Rica}

\section{Aspectos generales}

Las Cooperativas de Ahorro y Crédito (CAC) son entidades de carácter privado, de naturaleza cooperativa, que se constituyen con el propósito de promover el ahorro entre sus asociados y de crear, con el producto de esos recursos, una fuente de crédito que se les traslada a un costo razonable, para solventar sus necesidades. Asimismo para brindarles otros servicios financieros que funcionan mediante un esquema empresarial, que les permite administrar su propio dinero sobre la base de principios democráticos y mejorar sus condiciones sociales, económicas y culturales.

Es de interés social, la constitución y funcionamiento de estas organizaciones, como uno de los medios más eficaces para el desarrollo socioeconómico de los habitantes. ${ }^{11}$

Las Cooperativas de Ahorro y Crédito por disposición legal son fiscalizadas por la Superintendencia General de Entidades Financieras (SUGEF). ${ }^{12}$

La función de supervisión por lo general se efectúa a través de visitas y/o mediante de la revisión y el análisis de los informes emitidos por las mismas entidades, por lo que se requiere que las entidades publiquen la información financiera periódica.

En cuanto a la publicación de la información financiera, en Costa Rica, se dispone mediante el reglamento denominado "Acuerdo SUGEF 3-96 Normas para la Presentación, Remisión y Publicación de los Estados Financieros de las Entidades Sujetas a Fiscalización de la SUGEF», al igual que el resto de las entidades supervisadas, las CAC (Cooperativas de Ahorro y Crédito), deben publicar en un medio de comunicación escrito de circulación nacional, el balance de situación, estado de resultados y el dictamen de los auditores externos al cierre del ejercicio financiero anual, a más tardar el último día del mes de febrero, conforme a los formatos preestablecidos. En ese mismo plazo,

11 Ley de Regulación de la Actividad de Intermediación Financiera de las Organizaciones Cooperativas, Ley 7391, del 23 de abril de 1994.

12 Artículo 7 de la Ley Ibíd. 
las entidades fiscalizadas deberán publicar mediante su sitio WEB, los estados financieros completos: balance de situación, estado de resultados, estado de cambios en la situación financiera, estado de variaciones en el capital, así como las políticas contables utilizadas y las demás notas.

\section{Supervisión}

La supervisión de las CAC se establece mediante la Ley de Regulación de la Actividad de Intermediación Financiera de las Organizaciones Cooperativas, No. 7391, del 23 de abril de 1994 y supletoriamente Ley de Asociaciones Cooperativas, No. 6756 del 5 de mayo de 1982, y sus reformas, Ley Orgánica del Banco Central, No. 7558 y las disposiciones reglamentarias emitidas por el Consejo Nacional de Supervisión del Sistema Financiero.

La supervisión por parte de la SUGEF ha venido evolucionando hacia un sistema de supervisión más preventivo con el propósito de mantener sólido el sistema financiero. Las herramientas de supervisión extra situ —en la oficina - se basan en herramientas de monitoreo a través de sistemas estandarizados de indicadores o sets propios, ambos pueden, por ejemplo, "producir» una calificación o un ranking.

Este método de monitoreo está basado en otras experiencias principalmente en el CAMEL desarrollado por la Reserva Federal de Estados Unidos -y que en algunos países mantienen el nombre o agregan una "S» para reflejar la sensitividad para riesgos de mercado según la recomendación de Basilea de 1997- y varían los indicadores y los parámetros. Los cinco componentes del modelo SUGEF son: suficiencia patrimonial, utilidades, gestión, evaluación de la calidad de los activos, y flujo de fondos.

La evaluación de los componentes puede ser asignada por el analista financiero durante la revisión mensual de los estados financieros. Sin embargo, esta evaluación y calificación debe ser validada durante la visita de supervisión. Por disposición de la Ley Orgánica del Banco Central de Costa Rica se establece se juzga la situación económica y financiera de las entidades fiscalizadas con el propósito de velar por la estabilidad y la eficiencia del sistema financiero. ${ }^{13}$ Dicha disposición legal fue implementada en el Acuerdo SUGEF 24-00 REGLAMENTO PARA

13 Ley Orgánica del Banco Central de Costa Rica, No. 7558, del 3 de noviembre de 1995 y sus reformas. 

FISCALIZADAS, en el cual se califica el riesgo de la entidad supervisada.

Desde el punto de vista de las CAC los argumentos que hablan a favor de la supervisión:

Estímulo de la confianza pública y con esto, mayores posibilidades de crecer, así como una contribución a solucionar problemas de gobernabilidad que en muchos casos no hacen una disciplina financiera posible por fuerza propia.

La supervisión de las CAC está en función al tamaño de sus activos, según lo indica el Art.117 de la Ley Orgánica del Banco Central de Costa Rica y complementado con el acuerdo del Consejo Nacional de Supervisión del Sistema Financiero mediante Artículo 8 del Acta de la Sesión 347-2002, celebrada el 19 de diciembre del 2002, en el cual se dispuso que serán supervisadas aquellas CAC que sus activos superen los US $\$ 1.08 .0$ millones.

\section{Perspectivas de desarrollo para CAC supervisadas}

Con las nuevas tendencias de la globalización y de competencia abierta, las CAC deben ser supervisadas si quieren competir, ya que la fiscalización oficial da mayor confianza a los asociados y al público en general. Sin embargo, el proceso de la definitiva inclusión al mercado regulado tiende a ser muy exigente (capital mínimo, creación de estimaciones para incobrables, clasificación de cartera según el riesgo, reglas para la concesión de créditos e inversiones en actividades no financieras, planes de cuentas uniformes y envío de información oportuna a la Superintendencia).

Por el otro lado, en la medida en que las CAC estén integradas cada vez más en la supervisión especializada adquirirán también «derechos morales» frente a los Bancos Centrales tales como la posibilidad de tener en el futuro cuentas corrientes en los institutos emisores, como en el caso de Costa Rica de que pueden participar plenamente en el sistema de pagos que tiene el Banco Central de Costa Rica.

\section{Normativa prudencial}

Tal vez el punto de referencia clave de la normativa prudencial para intermediarios financieros es el capital. En el caso de Costa Rica, la SUGEF ha implementado los criterios de adecuación patrimonial 
para las CAC. Los porcentajes aplicados van en algunos países mucho más allá de los coeficientes.

En ningún caso se exige de las cooperativas menos que la banca, en muchos casos sin embargo una solvencia más alta. Se fundamenta sobre todo en el hecho que en el caso de tensiones financieras en una cooperativa no hay un inversionista estratégico disponible tal como puede ser el caso en una sociedad anónima. En este contexto, la solvencia exigida constituye un colchón.

Algunas regulaciones prudenciales a las CAC y al resto de entidades supervisadas por al SUGEF son las siguientes:

- Acuerdo SUGEF 1-95 Normas generales para la clasificación y calificación de los deudores de la cartera de crédito, según el riesgo y para la constitución de las estimaciones correspondientes.

- Acuerdo SUGEF 4-96 Reglamento para el otorgamiento de crédito a grupos de interés económico.

- Acuerdo SUGEF 22-00 Normas Generales para definir y calcular el Patrimonio de las Entidades Fiscalizadas por la Superintendencia General de Entidades Financieras.

- Acuerdo SUGEF 23-00 Normas para establecer la Suficiencia Patrimonial de las Entidades Fiscalizadas por la Superintendencia General de Entidades Financieras.

- Acuerdo SUGEF 24-00 Reglamento para juzgar la Situación Económica-Financiera de las Entidades Fiscalizadas.

\section{Tipo de monitoreo}

En los últimos años se ha hecho más notorio en la necesidad de incrementar los esfuerzos por mantener el sistema financiero sano, sólido y solvente y a raíz de las crisis bancarias vividas en la década pasada son fieles testigos de esta necesidad, por lo que la SUGEF ha adoptado una serie de estándares internacionales para efectos de supervisión, tales como los Acuerdos de Basilea sobre Capitales Mínimos (1988) y sobre el Control de Riesgos de Mercado (1996), los 25 Principios Básicos de una Supervisión Eficaz (1997), la introducción de las Normas Internacionales de Contabilidad (NIC) o normas contra el Lavado de Dinero (40 Recomendaciones del FATF-GAFI).

Sin embargo, durante muchos años cuando se hablaba de intermediación financiera sólo se refería a la banca tradicional, dejando por fuera a las cooperativas de ahorro y crédito, que también se dedi- 
can a dicha actividad: captan y colocan igual que otros tipos de intermediarios.

Nuestro país utiliza el modelo CAMELS, más o menos «tropicalizados» del mismo, donde el "sets propios» de indicadores reúnen una serie de las características del propio CAMEL(S), en el cual se incluyen como actores de revisión los siguientes:

a) Capital (solvencia, capitalización, patrimonio o adecuación de capital).

b) Activos (calidad de activos, riesgo de mercado, riesgo de crédito).

c) Gestión (manejo, gestión operacional, gerencia, organización, riesgo de gestión).

d) Rentabilidad (resultados, utilidades, evaluación rendimientos, desempeño).

e) Liquidez (manejo de fondos, gestión de liquidez).

Además con base en ellos se obtienen calificaciones globales para cada institución supervisada, las cuales varían en su denominación (algunas numéricas y otras alfabéticas), pero que al final conducen a determinar el nivel de riesgo en el que se encuentra ubicada la institución de acuerdo con los resultados de los indicadores.

Nuestro país incluye la "S», de Sensibilidad a riesgos de mercado, de los resultados de los indicadores para cada concepto, la SUGEF lo produce, como apoyo a la supervisión, cuando menos una calificación por entidad o un ranking de las CAC con periodicidad mensual, que en nuestro caso, son conocidos sólo por las entidades supervisadas.

La base o los datos para la emisión de los indicadores se obtienen, en su gran mayoría, en forma extra situ y en muy pocos casos en el terreno (este último es utilizado básicamente para recopilar información cualitativa), información que normalmente se actualiza en forma mensual.

\section{Datos enviados a las Superintendencias - supervisión extra situ}

La información que por obvias razones, es solicitada por la SUGEF, es la relacionada con los estados financieros (básicamente balance general, estado de resultados, estado de flujos de efectivo y estado de cambios en el patrimonio neto), los informes y dictámenes anuales de los auditores externos y los datos para el cálculo de la relación de sol- 
vencia (de patrimonio técnico a activos ponderados por riesgo), además de aquella información que se considera apropiada para evaluar los niveles de exposición de riesgos de las entidades, tales como los riesgos de crédito, de liquidez y de tasa de interés.

No se debe olvidar que la misma Cooperativa enfrenta la obligación de establecer una propia evaluación del riesgo en todas sus áreas de participación, garantizando tanto la participación adecuada de los asociados como la efectividad del servicio que se presta. Sin desmeritar claro está, la consecución de una gestión social integrada que permita el alcanzar los objetivos y resultados establecidos como órgano empresarial, pero en auxilio de la organización social de servicio y solidaridad que identifican y fortalecen a la organización cooperativa.

\section{Bibliografía}

1) Costa Rica, Constitución Política de la República de Costa Rica, 7 de noviembre de 1949.

2) Costa Rica, Ley Orgánica del Sistema Bancario Nacional, Ley No. 1644 del 26 de setiembre de 1953.

3) Costa RicA, Ley Orgánica del Banco Central, ley No. 7558 de 3 de noviembre de 1995, Alcance No. 55 a La Gaceta No. 225 de 27 de noviembre de 1995.

4) Costa RicA, Ley de Regulación de la Actividad de Intermediación Financiera de las Organizaciones Cooperativas, Ley No. 7391, del 27 de abril de 1994, Publicada en La Gaceta No. 99 de 24 de mayo de 1994 y sus últimas reformas: Ley No. 7558 de 3 de noviembre de 1995. Alcance a LG\# 225 de 27 de noviembre de 1995.

5) Costa RicA, Ley de Asociaciones Cooperativas y Creación del INFOCOOP No. 4179.

6) Consejo Nacional de Supervisión del Sistema Financiero, Acuerdo SugeF 1-95, Normas generales para la clasificación y calificación de los deudores de la cartera de crédito, según el riesgo y para la constitución de las estimaciones correspondientes, aprobado en Acta de la Sesión 193-2000, celebrada el 27 de noviembre del 2000. Publicado en el diario oficial La Gaceta 3, del 4 de enero del 2001 y sus reformas.

7) Consejo Nacional de Supervisión del Sistema Financiero, Acuerdo SugeF 4-96, Reglamento para el otorgamiento de crédito a grupos de interés económico, aprobado en Acta de Sesión 20-96, artículo 6, celebrada el 16 de julio de 1996. Publicado en el diario oficial La Gaceta 147, del 5 de agosto de 1996.

8) Consejo Nacional de Supervisión del Sistema Financiero, Acuerdo Sugef 22-00, Normas Generales para Definir y Calcular el Patrimonio de las Entidades Fis- 
calizadas por la Superintendencia General de Entidades Financieras, aprobado en acta de la sesión 197-2000, celebrada el 11 de diciembre del 2000, publicado en el diario oficial La Gaceta 6, del 9 de enero del 2001 y sus reformas.

9) Consejo Nacional de Supervisión del Sistema Financiero, Acuerdo Sugef 23-00, Normas para Establecer la Suficiencia Patrimonial de las Entidades Fiscalizadas por la Superintendencia General de Entidades Financieras, aprobado en acta de la sesión 197-2000, celebrada el 11 de diciembre del 2000, publicado en el diario oficial La Gaceta 6, del 9 de enero del 2001.

10) Consejo Nacional de Supervisión del Sistema Financiero, Acuerdo SugeF 24-00, Reglamento para Juzgar la Situación Económica-Financiera de las Entidades Fiscalizadas, aprobado Acta sesión 197-2000, celebrada el 11 de diciembre del 2000, publicado en el diario oficial La Gaceta 6, del 9 de enero del 2001 y sus reformas.

11) Kaplan, Alicia y Drimer, Bernardo, Las Cooperativas: Fundamentos - Historia - Doctrina, Argentina 1981. 3. ${ }^{a}$ edición.

12) Chaves Gómez, Alvaro. Los Organismos Auxiliares del Movimiento Cooperativo. CEDAL 1985. Costa Rica. 